\title{
Congenital Cardiovascular Abnormality
}

National Cancer Institute

\section{Source}

National Cancer Institute. Congenital Cardiovascular Abnormality. NCI Thesaurus. Code C35729.

A heart or vascular abnormality which is inborn or present at birth. 